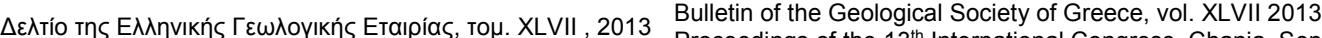

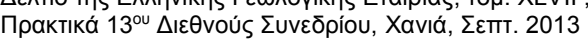
2013

\title{
GEOCHEMICAL EFFECT OF THE ROCK CHEMISTRY AND THE ANTHROPOGENIC ACTIVITIES ON GROUNDWATER: THE CASE STUDY OF NW EUBOEA, GREECE
}

\author{
Kanellopoulos C. ${ }^{1}$ and Mitropoulos $\mathbf{P} .^{2}$ \\ ${ }^{1}$ Doctor in Geological Sciences, 15 Pindou str., Vrilissia, Athens, 15235, Greece, \\ ckanellopoulos@gmail.com \\ ${ }^{2}$ Professor in Geochemistry, University of Athens, Department of Geology and Geoenvironment \\ Panepistimiopolis, Ilisia, 157 84, Athens, Greece,pmitrop@geol.uoa.gr
}

\begin{abstract}
A geochemical study of NW Euboea island ground waters was undertaken, in order to examine the possible effect of the chemical composition of the country rocks of the area as well as of the anthropogenic activities, to the concentration of environmentally important elements and chemical compounds in the groundwaters. $N W$ Euboea consists of a great variety of rock types showing a wide range in mineralogical and chemical composition. The main groups of rocks occurring in $N W$ Euboea are: i) various types of sedimentary rocks e.g. shale and chert formations, carbonate and clastic rocks, ii) ophiolitic rocks including peridotite, gabbro, serpentinite etc, and iii) epizonally metamorphosed basic igneous rocks, with schist and phyllite intercalations. A number of hot springs also occur in the area. The main anthropogenic activity in the area is the agricultural land use, as any significant industrial activity is absent. For that purpose, 45 water samples were collected and analyzed by Spectrophotometry for the main anions and by FP and AAS for major and a number of trace elements. On the basis of those analyses, a number of the water samples were selected and analyzed by ICP-AES and ICP-MS for a large group of mainly metallic trace elements. The interpretation of the analytical data showed clearly that the content of the groundwater for a significant group of trace elements (e.g. $\mathrm{Cr}, \mathrm{Ni}, \mathrm{Zn}$ ) was considerably influenced by the chemical composition of the surrounding rocks, especially the ophiolitic and metamorphic rocks. The anthropogenic activities also affect the groundwater quality, near areas where the use of fertilizers and pesticides for agricultural purposes is extensive, resulting to the increase of various anion concentrations $\left(\mathrm{NO}_{3}{ }^{-}, \mathrm{SO}_{4}^{-2}, \mathrm{PO}_{4}^{-3}\right)$.
\end{abstract}

Key words: groundwater geochemistry, trace element and anion concentration, $N W$ Euboea, Greece.

\section{Пєрі́ $\eta \psi \eta$}

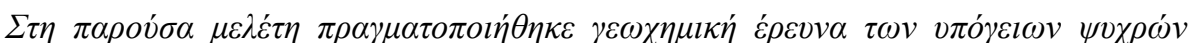

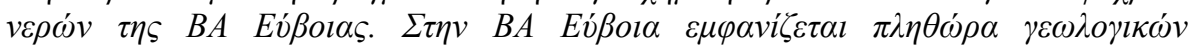

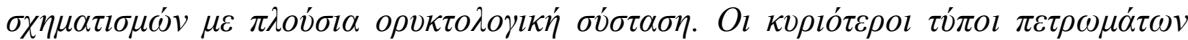

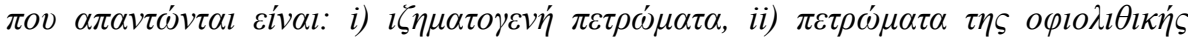

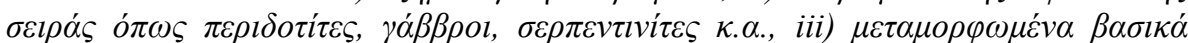




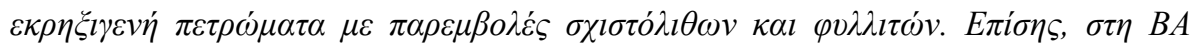

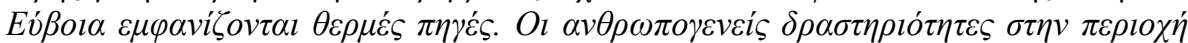

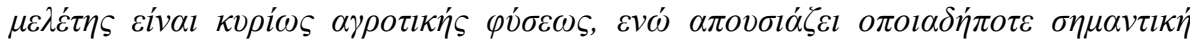

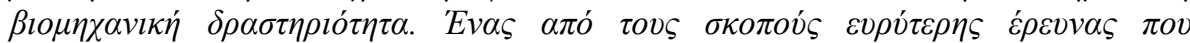

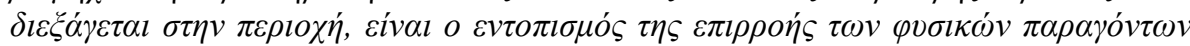

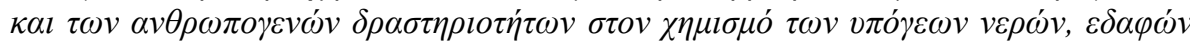

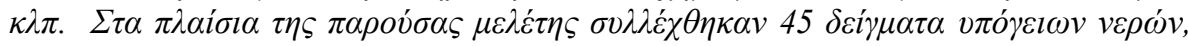

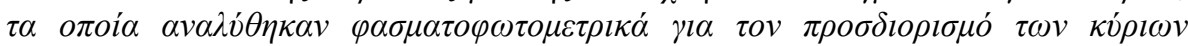

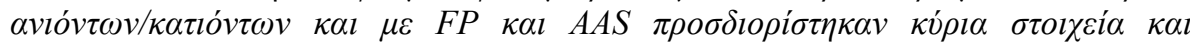

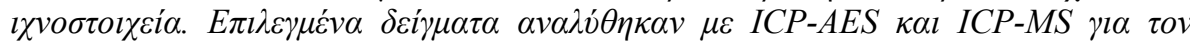

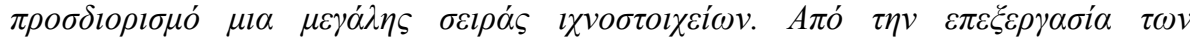

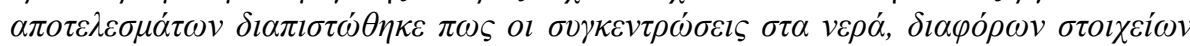

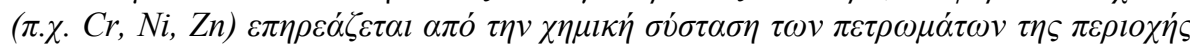

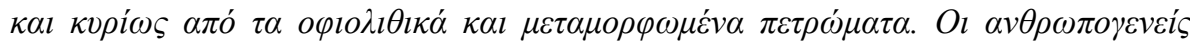

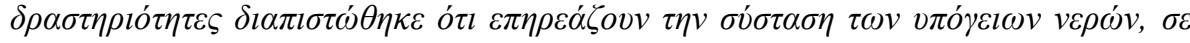

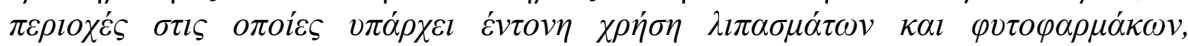

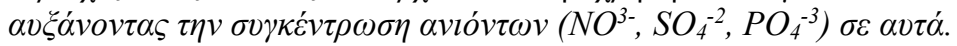

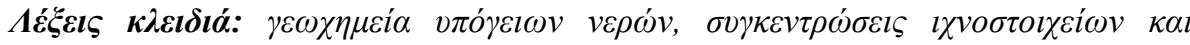
$\alpha v i o ́ v \tau \omega v, B \Delta E \dot{\beta} \beta o \imath \alpha, E \lambda \lambda \alpha ́ \delta \alpha$.

\section{Introduction}

Groundwater, plays a basic role in human life. The existence of sufficient groundwater resources and the maintenance of their quality is of major importance for the survival of man. It is thus indispensable, to protect these resources and ensure their sustainability. Environmental deterioration has led to a growing public concern over the potential accumulation of harmful elements and other contaminants in groundwater (Kabata-Pendias, 1995). The contamination of groundwater can pose long term environmental and health implications (Kabata-Pendias, 2007; Mueller, 1994; Needleman, 1980).

Water, whose chemical composition controls the circulation of element in ecosystems, is the main carrier of trace elements for vegetation. The prevailing opinion is that high concentrations of trace elements, which at many cases are potentially toxic, are linked with anthropogenic activities. In the present paper, a non-industrial area, where only small scale agricultural activities take place, was studied. At the study area there are occurrences of ultramafic rocks from the ophiolitic sequence. Many studies have proven that from the weathering of that type of rocks, triggers the enrichment of specific elements in nearby soils e.g. Co, Ni, Cr, As, Fe, Mn (Kanellopoulos and Argyraki, 2013; Oze et al., 2003; Gasser \& Dahlgren, 1994; Alexander et al., 1989; Gough et al. 1989; Brooks, 1987; Schreier et al., 1987).

The aim of this paper is to assess the impact of both anthropogenic and natural factors on the geochemistry of groundwater.

\section{Geological Setting}

Euboea Island is located in central Greece. The studied area lies between latitudes $38^{\circ} 58^{\prime}$ and $38^{\circ}$ $50.5^{\prime}$ and longitudes $22^{\circ} 49.5^{\prime}$ and $23^{\circ} 10.5^{\prime}$. It is characterized by rocky mountainous topography and some lowland areas.

The Northeastern part of Euboea island belongs geologically to the Pelagonian and SubPelagonian units, which form the western part of the internal geotectonic units of Greece (Mountrakis, 1986; Aubouin, 1959). 
The study area consists of non-metamorphic rocks of the ophiolitic series, including peridotite, gabbro and serpentinite, as well as of metamorphic rocks. Large parts of NW Euboea are covered by Post Alpine formations and sediments of Quaternary to Neogene age (Fig. 1).

In the centre of the northern Evoicos gulf, the volcanogenic islands of Lichades are located (Georgalas, 1938). They are made mainly of trachyandesite lava flows, dated at $0.5 \mathrm{Ma}$ (Pe-Piper and Piprer, 2002). The whole area is highly faulted due to extensional tectonics (Vavassis, 2001, Le Pichon and Angelier, 1979; McKenzie 1970; 1972). In the studied area many hot springs exist (Gioni-Stavropoulou, 1983), showing high concentrations of a large group of major and trace elements (Kanellopoulos, 2011; 2006). Finally in the areas of Loutra Edipsou and Ilia, thermogenic travertine deposits exist, created by the local hot-springs (Kanellopoulos, 2012; 2011).

\section{Materials and Methods}

\subsection{Groundwater Sampling and Analysis}

A total of 45 groundwater samples were collected (Table 1) from springs, wells and drills mainly used for agricultural activities and water supply of villages. Sensitive physicochemical parameters such as, $\mathrm{pH}$, Temperature, E.C., T.D.S. were measured in the field. All the samples were vacuum filtered, acidified to a final concentration of $2 \%$ nitric acid, stored in polyethylene bottles and preserved in a refrigerator.

All the 45 water samples were analyzed in the Laboratory of Economic Geology and Geochemistry, University of Athens. The anion concentrations were measured spectrophotometrically (bicarbonate was measured by titration) while the major and trace element concentrations were measured by Flame Photometry and Atomic Absorption Spectroscopy (AAS) (Table 2).

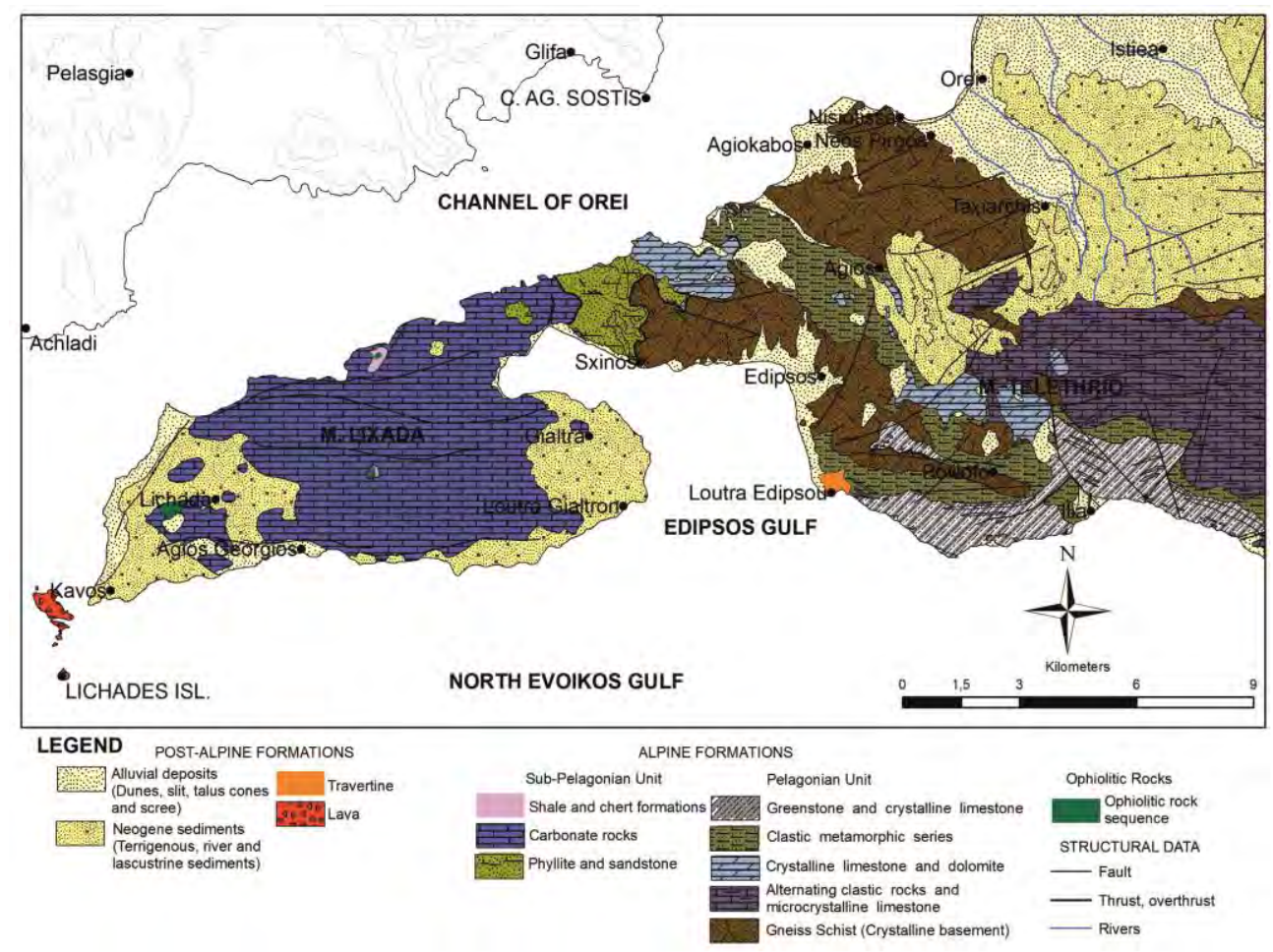

Figure 1 - Geological map of the study area (Kanellopoulos, 2011).

XLVII, No 2 - 944 
Table 1 - Samples locality, physicochemical parameters, hydrochemical type, SAR, EC, MH values and classification based on SAR-EC.

\begin{tabular}{|c|c|c|c|c|c|c|c|c|c|c|}
\hline Code & Locality & $\begin{array}{c}\text { Sampling site } \\
\text { (depth in m) }\end{array}$ & $\begin{array}{c}\mathrm{T} \\
\left({ }^{\circ} \mathrm{C}\right)\end{array}$ & pH & $\begin{array}{l}\text { TDS } \\
\text { (g/lt) }\end{array}$ & $\begin{array}{c}\mathrm{EC} * \\
(\mathrm{mS} / \mathrm{cm})\end{array}$ & $\begin{array}{l}\text { Hydroc. } \\
\text { type }\end{array}$ & $\begin{array}{c}\text { SAR-EC } \\
\text { classif. }\end{array}$ & SAR & MH \\
\hline AD-11 & Edipsos-Schinos & well (4) & 17.5 & 7.66 & 1.27 & 3.33 & $\mathrm{Ca}-\mathrm{Cl}$ & $\mathrm{C} 3-\mathrm{S} 1$ & 0.811 & 21.9 \\
\hline AD-22 & Edipsos-Schinos & well (4) & 21.9 & 7.85 & 1.77 & 3.55 & $\mathrm{Na}-\mathrm{Cl}$ & C3-S2 & 8.569 & 28.1 \\
\hline AD-10 & Edipsos-Schinos & well (7) & 23.2 & 7.1 & 3.63 & 7.26 & $\mathrm{Na}-\mathrm{Cl}$ & $\mathrm{C} 2-\mathrm{S} 1$ & 7.117 & 26.4 \\
\hline AD-16 & Edipsos-Well Ф-18 & well (10) & 16 & 6.8 & 0.33 & 0.66 & $\mathrm{Ca}-\mathrm{HCO}_{3}$ & C1-S1 & 0.938 & 31.8 \\
\hline AD-17 & Edipsos-Village & drill & 14.45 & 7.45 & 0.41 & 0.84 & $\mathrm{Ca}-\mathrm{HCO}_{3}$ & $\mathrm{C} 2-\mathrm{S} 1$ & 0.949 & 43.9 \\
\hline AD-18 & Edipsos-Old factory & drill (120) & 12.2 & 6.99 & 0.475 & 0.96 & $\mathrm{Ca}-\mathrm{HCO}_{3}$ & $\mathrm{C} 2-\mathrm{S} 1$ & 1.214 & 35 \\
\hline HL-2 & Ilia & spring & 15 & 7.9 & 0.26 & 0.54 & $\mathrm{Mg}-\mathrm{HCO}_{3}$ & $\mathrm{C} 1-\mathrm{S} 1$ & 0.284 & 51.2 \\
\hline HL-10 & Ilia & spring & 18 & 8.27 & 0.25 & 0.51 & $\mathrm{Ca}-\mathrm{HCO}_{3}$ & $\mathrm{C} 1-\mathrm{S} 1$ & 0.397 & 46.7 \\
\hline AD-7 & Polylofo-Vitsa & spring & 15.3 & 7.7 & 0.39 & 0.78 & $\mathrm{Ca}-\mathrm{HCO}_{3}$ & $\mathrm{C} 2-\mathrm{S} 1$ & 0.759 & 26.1 \\
\hline AD-8 & Polylofo- St. George M. & spring & 12.7 & 8.1 & 0.27 & 0.53 & $\mathrm{Mg}-\mathrm{HCO}_{3}$ & $\mathrm{C} 1-\mathrm{S} 1$ & 0.266 & 50.3 \\
\hline GIA-1 & Gialtra-3 springs & spring & 21.3 & 6.96 & 0.69 & 1.36 & $\mathrm{Na}-\mathrm{SO}_{4}$ & $\mathrm{C} 2-\mathrm{S} 1$ & 2.4 & 43.6 \\
\hline GIA-2 & Gialtra-Averof spring & spring & 17.7 & 7.23 & 0.64 & 1.28 & $\mathrm{Na}-\mathrm{NO}_{3}$ & $\mathrm{C} 2-\mathrm{S} 1$ & 2.252 & 42.4 \\
\hline GIA-3 & Gialtra & drill & 19.1 & 7.6 & 0.33 & 0.69 & $\mathrm{Ca}-\mathrm{HCO}_{3}$ & $\mathrm{C} 1-\mathrm{S} 1$ & 0.997 & 45.1 \\
\hline GIA-5 & Gialtra-Near Averof sp. & spring & 14.1 & 7.7 & 0.37 & 0.74 & $\mathrm{Ca}-\mathrm{HCO}_{3}$ & $\mathrm{C} 1-\mathrm{S} 1$ & 0.8 & 49.4 \\
\hline GIA-6 & Gialtra-3 springs & spring & 20.1 & 8.06 & 0.66 & 1.34 & $\mathrm{Ca}-\mathrm{HCO}_{3}$ & $\mathrm{C} 2-\mathrm{S} 1$ & 1.838 & 47.3 \\
\hline GIA-10 & Gialtra & spring & 18 & 7.84 & 0.31 & 0.62 & $\mathrm{Mg}-\mathrm{HCO}_{3}$ & $\mathrm{C} 1-\mathrm{S} 1$ & 0.562 & 54.6 \\
\hline AGG-1 & St. George vil.-Kamara & spring & 19.9 & 6.96 & 1.18 & 2.37 & $\mathrm{Na}-\mathrm{Cl}$ & $\mathrm{C} 3-\mathrm{S} 1$ & 5.725 & 52.6 \\
\hline AGG-2 & St. George village & spring & 22.2 & 7.84 & 1.2 & 2.4 & $\mathrm{Na}-\mathrm{Cl}$ & $\mathrm{C} 3-\mathrm{S} 1$ & 6.224 & 55.2 \\
\hline LIX-1 & Lichada & drill & 17.9 & 7.27 & 0.54 & 1.09 & $\mathrm{Mg}-\mathrm{HCO}_{3}$ & $\mathrm{C} 2-\mathrm{S} 1$ & 0.506 & 60.2 \\
\hline LIX-2 & Lichada & drill & 23 & 7.85 & 0.52 & 1.05 & $\mathrm{Mg}-\mathrm{HCO}_{3}$ & $\mathrm{C} 2-\mathrm{S} 1$ & 0.513 & 60.6 \\
\hline AG-1 & Agios & well (10) & 15.35 & 6.91 & 0.61 & 1.23 & $\mathrm{Ca}-\mathrm{HCO}_{3}$ & $\mathrm{C} 2-\mathrm{S} 1$ & 1.33 & 25.4 \\
\hline AD-12 & Agios-Platania & spring & 12.7 & 7.6 & 0.25 & 0.51 & $\mathrm{Ca}-\mathrm{HCO}_{3}$ & C1-S1 & 0.273 & 48 \\
\hline AD-13 & Agios-Old factory & drill (120) & 14.3 & 7.33 & 0.46 & 0.93 & $\mathrm{Ca}-\mathrm{HCO}_{3}$ & $\mathrm{C} 2-\mathrm{S} 1$ & 1.026 & 38.6 \\
\hline BAR-1 & Varvara & spring & 15 & 7.39 & 0.51 & 1.03 & $\mathrm{Ca}-\mathrm{HCO}_{3}$ & $\mathrm{C} 2-\mathrm{S} 1$ & 0.571 & 41.7 \\
\hline AK-1 & Agiokabos-Skepasti & spring & 16.35 & 7.3 & 0.41 & 0.83 & $\mathrm{Ca}-\mathrm{HCO}_{3}$ & $\mathrm{C} 2-\mathrm{S} 1$ & 1.444 & 44.5 \\
\hline AK-2 & Agiokabos-Xalasmata & drill & 10.5 & 7.43 & 0.37 & 0.74 & $\mathrm{Ca}-\mathrm{HCO}_{3}$ & C1-S1 & 1.183 & 49.1 \\
\hline AK-3 & Agiokabos-Restorant & drill (6) & 14.3 & 7.25 & 0.39 & 0.8 & $\mathrm{Ca}-\mathrm{HCO}_{3}$ & $\mathrm{C} 2-\mathrm{S} 1$ & 0.774 & 33.7 \\
\hline AK-4 & Agiokabos & drill (36) & 15.8 & 7.3 & 0.32 & 0.64 & $\mathrm{Ca}-\mathrm{HCO}_{3}$ & C1-S1 & 0.643 & 40.4 \\
\hline AK-6 & Agiokabos & drill (60) & 15.2 & 7.19 & 0.33 & 0.66 & $\mathrm{Ca}-\mathrm{HCO}_{3}$ & $\mathrm{C} 1-\mathrm{S} 1$ & 0.636 & 38.9 \\
\hline AK-7 & Agiokabos & drill (4) & 18.3 & 7.96 & 0.46 & 0.93 & $\mathrm{Ca}-\mathrm{HCO}_{3}$ & $\mathrm{C} 2-\mathrm{S} 1$ & 1.17 & 28 \\
\hline NIS-1 & Nisiotissa & well & 13.1 & 7.24 & 0.42 & 0.85 & $\mathrm{Ca}-\mathrm{HCO}_{3}$ & $\mathrm{C} 2-\mathrm{S} 1$ & 1.299 & 31.5 \\
\hline NIS-2 & Nisiotissa & well & 14.6 & 7.19 & 0.41 & 0.84 & $\mathrm{Ca}-\mathrm{HCO}_{3}$ & $\mathrm{C} 2-\mathrm{S} 1$ & 1.696 & 32.8 \\
\hline NIS-3 & Nisiotissa & drill & 18.25 & 6.86 & 0.41 & 0.83 & $\mathrm{Mg}-\mathrm{HCO}_{3}$ & $\mathrm{C} 2-\mathrm{S} 1$ & 1.404 & 51.9 \\
\hline NP-1 & N. Pirgos-Tsempetsis & well & 17.7 & 7.23 & 0.56 & 1.13 & $\mathrm{Ca}-\mathrm{HCO}_{3}$ & $\mathrm{C} 2-\mathrm{S} 1$ & 2.19 & 41.2 \\
\hline NP-3 & N. Pirgos-Lowland & drill (6) & 17.5 & 7.45 & 0.53 & 1.07 & $\mathrm{Ca}-\mathrm{HCO}_{3}$ & $\mathrm{C} 2-\mathrm{S} 1$ & 0.712 & 45.7 \\
\hline NP-4 & N. Pirgos-Lowland & drill (9) & 15.6 & 7.3 & 0.48 & 0.93 & $\mathrm{Ca}-\mathrm{HCO}_{3}$ & $\mathrm{C} 2-\mathrm{S} 1$ & 0.837 & 41.6 \\
\hline NP-5 & N. Pirgos-Arida & drill (50) & 16.3 & 7.29 & 0.59 & 1.17 & $\mathrm{Ca}-\mathrm{HCO}_{3}$ & $\mathrm{C} 2-\mathrm{S} 1$ & 0.763 & 43.1 \\
\hline NP-6 & $\begin{array}{c}\text { N. Pirgos-New } \\
\text { aqueduct }\end{array}$ & drill (60) & 16.6 & 7.37 & 0.38 & 0.77 & $\mathrm{Ca}-\mathrm{HCO}_{3}$ & $\mathrm{C} 1-\mathrm{S} 1$ & 0.885 & 38.4 \\
\hline NP-7 & N. Pirgos-Old aqueduct & drill (60) & 16.6 & 7.36 & 0.9 & 0.44 & $\mathrm{Ca}-\mathrm{HCO}_{3}$ & $\mathrm{C} 2-\mathrm{S} 1$ & 0.852 & 44.1 \\
\hline NP-8 & N. Pirgos-New church & well (3) & 12.15 & 7.76 & 0.28 & 0.56 & $\mathrm{Ca}-\mathrm{HCO}_{3}$ & $\mathrm{C} 1-\mathrm{S} 1$ & 0.428 & 31.4 \\
\hline NP-9 & N. Pirgos-Old church & drill & 14.4 & 7.39 & 0.41 & 0.82 & $\mathrm{Ca}-\mathrm{HCO}_{3}$ & $\mathrm{C} 2-\mathrm{S} 1$ & 0.576 & 44 \\
\hline NP-10 & N. Pirgos-Kolonaki & well (5) & 14.1 & 7.13 & 0.42 & 0.86 & $\mathrm{Ca}-\mathrm{HCO}_{3}$ & $\mathrm{C} 2-\mathrm{S} 1$ & 1.278 & 47.4 \\
\hline NP-11 & N. Pirgos-Kolonaki & well (5) & 14.4 & 6.95 & 0.35 & 0.72 & $\mathrm{Ca}-\mathrm{HCO}_{3}$ & C1-S1 & 1.414 & 44.1 \\
\hline OR-1 & Oreoi-School & spring & 10.75 & 7.72 & 0.38 & 0.76 & $\mathrm{Ca}-\mathrm{HCO}_{3}$ & C1-S1 & 0.625 & 49.8 \\
\hline
\end{tabular}


Table 2 - Concentrations of chemical parameters analyzed by AAS, FP, SP and titration.

\begin{tabular}{|c|c|c|c|c|c|c|c|c|c|c|c|c|c|c|c|c|c|}
\hline & Cd & Co & $\mathrm{Cr}$ & Mn & $\mathbf{P b}$ & $\mathbf{N i}$ & $\mathrm{Fe}$ & Zn & $\mathrm{Na}$ & K & Mg & $\mathrm{Ca}$ & $\mathrm{PO}_{4}$ & $\mathrm{NO}_{3}$ & $\mathrm{SO}_{4}$ & Cl & HCO \\
\hline & $\mu \mathrm{g} / \mathrm{L}$ & $\mu \mathrm{g} / \mathrm{L}$ & $\mu \mathrm{g} / \mathrm{L}$ & $\mu \mathrm{g} / \mathrm{L}$ & $\mu \mathrm{g} / \mathrm{L}$ & $\mu \mathrm{g} / \mathrm{L}$ & $\mu \mathrm{g} / \mathrm{L}$ & $\mu \mathrm{g} / \mathrm{L}$ & $\mathrm{mg} / \mathrm{L}$ & $\mathrm{mg} / \mathrm{L}$ & $\mathrm{mg} / \mathrm{L}$ & $\mathrm{mg} / \mathrm{L}$ & $\mathrm{mg} / \mathrm{L}$ & $\mathrm{mg} / \mathrm{L}$ & $\mathrm{mg} / \mathrm{L}$ & $\mathrm{mg} / \mathrm{L}$ & $\mathrm{mg} / \mathrm{L}$ \\
\hline AD-11 & 0.1 & 1.8 & $<0.2$ & 9 & $<1$ & 3 & 22 & 420 & 42 & 8 & 34.1 & 200 & 0.21 & 9.2 & 160 & 650 & 320 \\
\hline AD-22 & $<0.1$ & $<0.2$ & 0.3 & 1 & $<1$ & $<0.6$ & 9 & \begin{tabular}{|l|}
150 \\
\end{tabular} & 465 & 5 & 38.1 & 160.5 & 0.23 & 9.7 & 200 & 848 & 295 \\
\hline AD-10 & 0.3 & 0.3 & 0.3 & 0.6 & $<1$ & $<0.6$ & 2 & 5 & - & - & - & - & 0.353 & 1 & 70 & 410 & 283 \\
\hline AD-16 & $<0.1$ & $<0.2$ & 0.9 & 0.6 & $<1$ & $<0.6$ & 7 & 10 & 34 & 3 & 19.2 & 68 & 0.25 & 9.7 & 39 & 36 & 280 \\
\hline AD-17 & $<0.1$ & $<0.2$ & 0.4 & 1 & $<1$ & 0.6 & 2 & 34 & 39 & 4 & 34.1 & 72 & 0.14 & 29 & 70 & 38 & 313 \\
\hline AD-18 & $<0.1$ & $<0.2$ & 0.9 & 0.7 & $<1$ & $<0.6$ & 3 & 10 & 51 & 4 & 28.4 & 87 & 0.24 & 19.8 & 26 & 66 & 381 \\
\hline HL-2 & $<0.1$ & 0.5 & 0.4 & 0.8 & $<1$ & $<0.6$ & 3 & 9 & 10 & 3 & 29.3 & 46 & 0.13 & 7 & 20 & 9.9 & 154 \\
\hline HL-10 & - & - & - & - & - & - & - & - & - & - & - & - & 0.198 & 0.5 & 38.6 & 2.41 & 213 \\
\hline AD-7 & $<0.1$ & 1.5 & $<0.2$ & $<0.5$ & $<1$ & $<0.6$ & 4 & 17 & 24 & 5 & 12 & 56 & 0.12 & 24.2 & 30 & 20.2 & 292 \\
\hline AD-8 & 0.4 & 1.3 & 0.3 & $<0.5$ & $<1$ & $<0.6$ & 8 & 12 & 9 & 5 & 26.4 & 43 & 0.04 & 7 & 19 & 13 & 224 \\
\hline GIA-1 & $<0.1$ & 0.6 & 1 & $<0.5$ & $<1$ & $<0.6$ & 15 & 10 & 107 & 24 & 39.9 & 85 & 0.25 & 225.5 & 620 & 78 & 272 \\
\hline GIA-2 & $<0.1$ & 1.3 & 1 & $<0.5$ & $<1$ & $<0.6$ & 12 & 6 & 97 & 30 & 36.2 & 81 & 0.15 & 250.8 & 95 & 84 & 240 \\
\hline GIA-3 & $<0.1$ & 0.6 & 6 & 1 & $<1$ & 0.8 & 12 & 47 & 40 & 7 & 33.4 & 67 & 0.17 & 166.1 & 41 & 44 & 198 \\
\hline GIA-5 & $<0.1$ & 2.9 & 0.7 & 1 & $<1$ & $<0.6$ & 10 & 23 & 30 & 6 & 32 & 54 & 0.27 & 15 & 37 & 21.3 & 255 \\
\hline GIA-6 & $<0.1$ & $<0.2$ & 0.6 & $<0.5$ & $<1$ & $<0.6$ & 3 & $<2$ & 81 & 19 & 42.3 & 77.6 & 0.28 & 217.4 & 80 & 140.8 & 283 \\
\hline \begin{tabular}{|c|} 
GIA- \\
10 \\
\end{tabular} & - & - & - & - & - & - & - & - & - & - & - & - & 0.121 & 0.8 & 26.2 & 27.5 & 267 \\
\hline $\begin{array}{c}\text { AGG- } \\
1\end{array}$ & $<0.1$ & $<0.2$ & 0.2 & 1 & $<1$ & $<0.6$ & 3 & 4 & 275 & 9 & 55.8 & 83 & 0.1 & 7 & 80 & 478 & 330 \\
\hline \begin{tabular}{|c|} 
AGG- \\
2
\end{tabular} & $<0.1$ & $<0.2$ & 0.3 & $<0.5$ & $<1$ & $<0.6$ & 3 & $<2$ & 290 & 9 & 55.1 & 73.8 & 0.09 & 4.4 & 74 & 604 & 258 \\
\hline LIX-1 & - & - & - & - & - & - & - & - & - & - & - & - & 0.446 & 15.5 & 43 & 7.57 & 347 \\
\hline LIX-2 & - & - & - & - & - & - & - & - & - & - & - & - & 0.514 & 8.9 & 42.9 & 15.45 & 343 \\
\hline AG-1 & $<0.1$ & 0.2 & 0.2 & 120 & $<1$ & $<0.6$ & 2 & 5 & 60 & 13 & 23.8 & 115 & 3.04 & 73.5 & 94 & 64 & 298 \\
\hline AD-12 & $<0.1$ & $<0.2$ & $<0.2$ & $<0.5$ & $<1$ & $<0.6$ & 4 & 8 & 9 & 5 & 24.1 & 43 & 0.04 & 7 & 20 & 8.3 & 234 \\
\hline AD-13 & $<0.1$ & 0.5 & $<0.2$ & $<0.5$ & $<1$ & $<0.6$ & 3 & 13 & 42 & 6 & 29.8 & 78 & 0.17 & 18 & 34 & 60 & 352 \\
\hline BAR-1 & $<0.1$ & $<0.2$ & 0.4 & 0.9 & $<1$ & 0.9 & 2 & 3 & 27 & 4 & 42.9 & 99 & 0.32 & 29 & 55 & 52 & 410 \\
\hline AK-1 & $<0.1$ & 0.2 & $<0.2$ & 12 & $<1$ & $<0.6$ & 4 & 51 & 55 & 4 & 29.7 & 61 & 0.15 & 5.7 & 37 & 74 & 292 \\
\hline AK-2 & 0.2 & 0.9 & 0.3 & 0.5 & $<1$ & 0.6 & 2 & 46 & 43 & 4 & 29.8 & 51 & 0.2 & 5.3 & 29 & 42 & 300 \\
\hline AK-3 & 0.3 & $<0.2$ & $<0.2$ & 3 & $<1$ & $<0.6$ & 1 & 3510 & 32 & 3 & 26.5 & 86 & 0.15 & 6.2 & 46 & 28 & 365 \\
\hline AK-4 & $<0.1$ & $<0.2$ & $<0.2$ & 17 & $<1$ & 0.8 & 3 & 30 & 24 & 3 & 25.9 & 63 & 0.12 & 6.6 & 34 & 40 & 270 \\
\hline AK-6 & $<0.1$ & 0.4 & 0.4 & 1.8 & $<1$ & $<0.6$ & 2 & 8 & 24 & 4 & 25.5 & 66 & 0.06 & 7 & 40 & 28 & 285 \\
\hline AK-7 & $<0.1$ & $<0.2$ & $<0.2$ & 3 & $<1$ & 2 & 2 & 50 & 44 & 3 & 18.2 & 77.2 & 0.1 & 5.7 & 54 & 67.2 & 347 \\
\hline NIS-1 & $<0.1$ & $<0.2$ & 0.3 & 2 & $<1$ & $<0.6$ & 4 & 6 & 50 & 3 & 21.5 & 77 & 1.37 & 18.5 & 80 & 54 & 230 \\
\hline NIS-2 & $<0.1$ & $<0.2$ & 0.9 & 0.5 & $<1$ & $<0.6$ & $<2$ & 6 & 61 & 3 & 19.5 & 66 & 1.11 & 7.5 & 64 & 76 & 270 \\
\hline NIS-3 & $<0.1$ & 0.5 & 0.3 & 20 & $<1$ & $<0.6$ & 3 & 6 & 53 & 4 & 34 & 52 & 0.65 & 9.7 & 22 & 88 & 230 \\
\hline NP-1 & $<0.1$ & 0.7 & 0.6 & 80 & $<1$ & 5 & 7 & 15 & 88 & 7 & 30.6 & 72 & 2.02 & 40.5 & 44 & 72 & 386 \\
\hline NP-3 & \begin{tabular}{|l|}
$<0.1$ \\
\end{tabular} & $<0.2$ & 0.3 & 14 & $<1$ & $<0.6$ & 9 & 47 & 34 & 6 & 47.9 & 94 & 0.24 & 11.9 & 102.5 & 22.4 & 430 \\
\hline NP-4 & $<0.1$ & 0.6 & 3 & 1.3 & $<1$ & $<0.6$ & 6 & 49 & 36 & 6 & 35.4 & 82 & 0.39 & 18 & 39 & 28 & 366 \\
\hline NP-5 & $<0.1$ & 0.3 & 3 & 1.5 & $<1$ & $<0.6$ & 7 & 47 & 36 & 6 & 44.1 & 96 & 0.44 & 33.9 & 80 & 34 & 426 \\
\hline NP-6 & $<0.1$ & $<0.2$ & $<0.2$ & $<0.5$ & $<1$ & $<0.6$ & 7 & 22 & 33 & 6 & 24.6 & 65 & 0.19 & 10.1 & 25 & 20 & 277 \\
\hline NP-7 & $<0.1$ & $<0.2$ & 3 & 1 & $<1$ & 0.6 & 13 & 11 & 37 & 6 & 38.2 & 80 & 0.22 & 15.8 & 40 & 13.1 & 378 \\
\hline NP-8 & $<0.1$ & 0.2 & 2 & 0.9 & $<1$ & 1 & 3 & 10 & 15 & 5 & 17.8 & 64 & 0.3 & 22.9 & 35 & 13 & 249 \\
\hline NP-9 & $<0.1$ & $<0.2$ & 3 & 0.8 & $<1$ & $<0.6$ & $<2$ & 9 & 25 & 4 & 38.1 & 80 & 0.2 & 13.2 & 37 & 37 & 242 \\
\hline NP-10 & $<0.1$ & 0.2 & 0.4 & 1.6 & $<1$ & $<0.6$ & 3 & 4 & 50 & 4 & 33.4 & 61 & 0.43 & 57.6 & 52 & 54 & 200 \\
\hline NP-11 & $<0.1$ & $<0.2$ & 0.3 & 0.7 & $<1$ & $<0.6$ & 13 & 4 & 50 & 7 & 25.4 & 53 & 0.63 & 31.2 & 55 & 46 & 230 \\
\hline \begin{tabular}{|l|} 
OR-1 \\
\end{tabular} & $<0.1$ & 0.3 & 0.3 & 260 & $<1$ & 1 & 3 & 19 & 27 & 7 & 42.7 & 71 & 0.18 & 4.8 & 2 & 20.1 & 380 \\
\hline P.V. & 5 & & 50 & & 10 & 20 & & & & & & & & 50 & & & \\
\hline
\end{tabular}

XLVII, No 2 - 946 
On the basis of the above analytical data, 11 of the water samples were analyzed by Inductively Coupled Plasma-Atomic Emission Spectroscopy (ICP-AES) and by Inductively Coupled PlasmaMass Spectrometry (ICP-MS) at the ACME Analytical Laboratories Ltd., Canada, for a series of elements (Table 3).

Detailed description of the used analytical methods as well as, the analytical quality control procedures, including analysis of blank and duplicate samples as well simultaneous analysis of Certified Reference water samples, are given in Kanellopoulos, 2011.

\section{Analytical Results}

\subsection{Chemical Analysis}

The location of the samples is presented in Table 1, while in Tables 1,2 and 3 , the physicochemical parameters analysed both in situ and in the lab, are presented.

In Figure 2A, the chemical analyses were plotted in the Piper diagram, in order to evaluate the hydrochemistry of the studied groundwater samples. In that Piper diagram, 39 of the studied groundwater samples are plotted in the same area and most of them have $\mathrm{Ca}-\mathrm{HCO}_{3}$ hydrochemical type (Table 1). From that group, only four samples have different hydrochemical type, as a result of the differentiation of the local bedrocks (Lichada area: LIX-1, LIX-2, Ilia area: HL-2, AD-8).

From all the studied samples only 6 are plotted separately due to their differed hydrochemical type, reflecting the impact of specific factors controlling the chemistry of the samples, like intrusion of sea water (AD-10, AD-11, AD-22, AGG-1 and AGG-2, hydr. type Na-Cl) or impact of fertilizers (GIA-1, hydr. type $\mathrm{Na}_{-} \mathrm{SO}_{4}$ ). All the samples with $\mathrm{Na}-\mathrm{Cl}$ hydrological type are from shallow aquifers located at a short distance from the shoreline $(10-20 \mathrm{~m})$ and present high concentration of $\mathrm{Cl}$ (Table 2).

In order to visualize the spatial relationship between the concentrations and the geological features in the studied area, graduated symbol maps were created by plotting the results of the chemical analysis. Class intervals were selected on the basis of the statistical distribution for each element and appear with different sized symbol on the maps, representing the following concentration classes: (a) minimum- $1^{\text {st }}$ quartile, (b) $1^{\text {st }}$ quartile- median (baseline concentration), (c) median- $3^{\text {rd }}$ quartile and $(d)>3^{\text {rd }}$ quartile- maximum. These maps are presented in Figures $2 \mathrm{E}-2 \mathrm{H}$.

The concentrations of $\mathrm{Na}$ and $\mathrm{Cl}$ vary in the same way (Fig. 2B), suggesting common source. The samples with higher concentrations of $\mathrm{Cl}(848 \mathrm{mg} / \mathrm{L})$ and $\mathrm{Na}(642 \mathrm{mg} / \mathrm{L})$ are from shallow aquifers located at short distance from the sea. For instance, samples AD-11, AD-22 and AD-10 were taken from shallow wells near the seashore at Schinos area (Fig. 2E).

The concentrations of $\mathrm{K}, \mathrm{NO}_{3}, \mathrm{SO}_{4}, \mathrm{PO}_{4}$ also co-vary satisfactorily (Fig. C), suggesting common source. The relation coefficient between $\mathrm{K}$ and $\mathrm{NO}_{3}$ is 0.86 . High concentrations are presented in samples located near lowlands, where agricultural activities take place (Fig. 2F).

Some samples present very high concentrations of $\mathrm{NO}_{3}$ as compared to $\mathrm{SO}_{4}, \mathrm{PO}_{4}$ and $\mathrm{K}$ concentrations (e.g. GIA-1, GIA-2, GIA-3, AG-1 etc). These samples are located within or are adjacent to villages without sewerage.

Concentrations of Ni, Mg, Zn (Fig. 2G) and to a smaller extent Fe, Cr, Co show high values near the crystalline basement. These areas are mostly lowlands, usually covered by Post-Alpine formations, characteristic examples are the areas of Neos Pyrgos and Agiokampos. Also, the concentrations of $\mathrm{Cr}$ and $\mathrm{Zn}$ co-varies (Fig. 2D). Of the above elements, $\mathrm{Zn}$ shows some outlier values $(>150 \mu \mathrm{g} / \mathrm{L})$ like the AK-3 sample $(3510 \mu \mathrm{g} / \mathrm{L})$. 
Table 3 - Concentrations of elements analyzed by ICP-MS.

\begin{tabular}{|c|c|c|c|c|c|c|c|c|c|c|c|c|}
\hline & & LIX-1 & LIX-2 & AGG-1 & GIA-1 & GIA-10 & AD-10 & HL-10 & AD-18 & AK-3 & NIS-3 & NP-7 \\
\hline $\mathbf{A g}$ & $(\mu \mathrm{g} / \mathrm{L})$ & - & - & $<0.05$ & $<0.05$ & - & - & - & $<0.05$ & $<0.05$ & $<0.05$ & $<0.05$ \\
\hline Al & $(\mu \mathrm{g} / \mathrm{L})$ & $<100$ & $<100$ & 8 & 7 & $<100$ & $<100$ & $<100$ & 10 & 5 & 9 & 6 \\
\hline As & $(\mu \mathrm{g} / \mathrm{L})$ & $<1000$ & $<1000$ & 2.6 & 4.2 & $<1000$ & $<1000$ & $<1000$ & 0.5 & $<0.5$ & $<0.5$ & 0.7 \\
\hline $\mathbf{A u}$ & $(\mu \mathrm{g} / \mathrm{L})$ & - & - & $<0.05$ & $<0.05$ & - & - & - & $<0.05$ & $<0.05$ & $<0.05$ & $<0.05$ \\
\hline B & $(\mu \mathrm{g} / \mathrm{L})$ & - & - & 193 & 409 & - & - & - & $<20$ & 91 & 25 & 48 \\
\hline $\mathbf{B a}$ & $(\mu \mathrm{g} / \mathrm{L})$ & 166 & 165 & 26 & 124 & 89 & 108 & 68 & 258 & 92 & 29 & 79 \\
\hline $\mathrm{Be}$ & $(\mu \mathrm{g} / \mathrm{L})$ & $<0.11$ & $<0.11$ & - & - & $<0.11$ & $<0.11$ & $<0.11$ & - & - & - & - \\
\hline $\mathrm{Br}$ & $(\mu \mathrm{g} / \mathrm{L})$ & - & - & 1692 & 242 & - & - & - & 134 & 136 & 303 & 100 \\
\hline $\mathbf{C a}$ & $(\mathrm{mg} / \mathrm{L})$ & 83.1 & 82.4 & 113.6 & 117.1 & 56.6 & 454.2 & 51.5 & 120 & 109.6 & 67.2 & 103 \\
\hline Cd & $(\mu \mathrm{g} / \mathrm{L})$ & 0.07 & 0.11 & $<0.05$ & $<0.05$ & $<0.05$ & $<0.05$ & 0.11 & $<0.05$ & 0.29 & $<0.05$ & $<0.05$ \\
\hline $\mathrm{Ce}$ & $(\mu \mathrm{g} / \mathrm{L})$ & $<0.24$ & $<0.24$ & 0.07 & 0.06 & $<0.24$ & $<0.24$ & $<0.24$ & 0.06 & 0.06 & 0.06 & 0.04 \\
\hline $\mathrm{Cl}$ & $\mathrm{mg} / \mathrm{L})$ & - & - & 522 & 102 & - & - & - & 88 & 46 & 113 & 35 \\
\hline Co & $(\mu \mathrm{g} / \mathrm{L})$ & $<0.09$ & $<0.09$ & 0.15 & 0.18 & $<0.09$ & 0.23 & $<0.09$ & 0.21 & 0.14 & 0.16 & 0.15 \\
\hline $\mathrm{Cr}$ & $(\mu \mathrm{g} / \mathrm{L})$ & 14 & 14 & $<0.5$ & 0.7 & $<4$ & 7 & $<4$ & 1.1 & $<0.5$ & $<0.5$ & 2.4 \\
\hline Cs & $(\mu \mathrm{g} / \mathrm{L})$ & $<0.09$ & $<0.09$ & 13.38 & 0.09 & $<0.09$ & $<0.09$ & $<0.09$ & 0.03 & $<0.01$ & 0.03 & $<0.01$ \\
\hline $\mathbf{C u}$ & $(\mu \mathrm{g} / \mathrm{L})$ & 3 & 3 & 1.8 & 2.8 & 3 & 5 & 2 & 4.1 & 1.4 & 1.1 & 2.3 \\
\hline $\mathbf{F e}$ & $(\mu \mathrm{g} / \mathrm{L})$ & $<100$ & $<100$ & 600 & 520 & $<100$ & $<100$ & $<100$ & 570 & 500 & 420 & 520 \\
\hline $\mathbf{G a}$ & $(\mu \mathrm{g} / \mathrm{L})$ & $<0.4$ & $<0.4$ & - & - & $<0.4$ & $<0.4$ & $<0.4$ & - & - & - & - \\
\hline Ge & $(\mu \mathrm{g} / \mathrm{L})$ & $<2$ & $<2$ & - & - & $<2$ & $<2$ & $<2$ & - & - & - & - \\
\hline $\mathbf{H g}$ & $(\mu \mathrm{g} / \mathrm{L})$ & - & - & 0.4 & $<0.1$ & - & - & - & 0.2 & 0.4 & 0.3 & $<0.1$ \\
\hline $\mathbf{K}$ & $(\mu \mathrm{g} / \mathrm{L})$ & 2630 & 2720 & 8130 & 21380 & 2250 & 9290 & 940 & 1140 & 560 & 950 & 1430 \\
\hline La & $(\mu \mathrm{g} / \mathrm{L})$ & $<0.58$ & $<0.58$ & 0.06 & 0.05 & $<0.58$ & $<0.58$ & $<0.58$ & 0.19 & 0.07 & 0.08 & 0.04 \\
\hline $\mathbf{L i}$ & $(\mu \mathrm{g} / \mathrm{L})$ & 13.9 & 14.1 & 21.5 & 9.2 & 6.9 & 18.8 & 3.6 & 5.6 & 5.7 & 9.2 & 5 \\
\hline Lu & $(\mu \mathrm{g} / \mathrm{L})$ & $<0.001$ & $<0.001$ & $<0.01$ & $<0.01$ & $<0.001$ & $<0.001$ & $<0.001$ & $<0.01$ & $<0.01$ & $<0.01$ & 0.01 \\
\hline Mg & $(\mathrm{mg} / \mathrm{L})$ & 76.2 & 76.9 & 61.8 & 45 & 41.3 & 99 & 27.4 & 31.2 & 29 & 36.4 & 43.1 \\
\hline Mn & $(\mu \mathrm{g} / \mathrm{L})$ & $<10$ & $<10$ & 1.36 & 0.29 & $<10$ & $<10$ & $<10$ & 0.37 & 3.77 & 23.94 & 1.28 \\
\hline Mo & $(\mu \mathrm{g} / \mathrm{L})$ & $<5$ & $<5$ & 1.2 & 0.3 & $<5$ & $<5$ & $<5$ & 0.6 & 0.6 & 0.1 & 1.2 \\
\hline $\mathbf{N a}$ & $(\mathrm{mg} / \mathrm{L})$ & 26.6 & 26.9 & 286.4 & 86 & 22.8 & 642.1 & 14.2 & 58.1 & 30.1 & 52.8 & 23.7 \\
\hline $\mathbf{N b}$ & $(\mu \mathrm{g} / \mathrm{L})$ & $<0.3$ & $<0.3$ & - & - & $<0.3$ & $<0.3$ & $<0.3$ & - & - & - & - \\
\hline $\mathbf{N i}$ & $(\mu \mathrm{g} / \mathrm{L})$ & 4.9 & 4.4 & $<0.2$ & $<0.2$ & 1.5 & 6.1 & 1.2 & 0.4 & $<0.2$ & 0.6 & 0.3 \\
\hline $\mathbf{P}$ & $(\mu \mathrm{g} / \mathrm{L})$ & $<1000$ & $<1000$ & 44 & 79 & $<1000$ & $<1000$ & $<1000$ & 41 & 41 & 41 & 66 \\
\hline $\mathbf{P b}$ & $(\mu \mathrm{g} / \mathrm{L})$ & 0.8 & $<0.4$ & 0.1 & $<0.1$ & 0.5 & 0.4 & $<0.4$ & $<0.1$ & 0.6 & $<0.1$ & 0.1 \\
\hline $\mathbf{R b}$ & $(\mu \mathrm{g} / \mathrm{L})$ & 0.53 & 0.54 & 7.36 & 1.53 & 1.53 & 4.97 & 0.38 & 0.75 & 0.11 & 0.1 & 0.25 \\
\hline $\mathbf{S}$ & $\mathrm{mg} / \mathrm{L})$ & 9.61 & 9.6 & 21 & 24 & 6.34 & 94.6 & 8.02 & 7 & 12 & 6 & 11 \\
\hline $\mathbf{S b}$ & $(\mu \mathrm{g} / \mathrm{L})$ & $<0.2$ & $<0.2$ & $<0.05$ & 0.07 & $<0.2$ & $<0.2$ & $<0.2$ & 0.21 & 0.08 & $<0.05$ & 0.09 \\
\hline $\mathrm{Se}$ & $(\mu \mathrm{g} / \mathrm{L})$ & - & - & 10.1 & 1.6 & - & - & - & 2.3 & 0.8 & 1.7 & 0.9 \\
\hline $\mathbf{S i}$ & $(\mu \mathrm{g} / \mathrm{L})$ & 16100 & 16000 & 4200 & 13400 & 11800 & 10500 & 5000 & 12600 & 7600 & 13800 & 11000 \\
\hline Sn & $(\mu \mathrm{g} / \mathrm{L})$ & $<0.8$ & $<0.8$ & 0.15 & 0.25 & $<0.8$ & $<0.8$ & $<0.8$ & 0.31 & 0.21 & 1.15 & 1.71 \\
\hline $\mathrm{Sr}$ & $(\mu \mathrm{g} / \mathrm{L})$ & 362 & 360 & 368 & 222 & 315 & 2599 & 240 & 324 & 264 & 204 & 289 \\
\hline Ta & $(\mu \mathrm{g} / \mathrm{L})$ & $<0.03$ & $<0.03$ & - & - & $<0.03$ & $<0.03$ & $<0.03$ & - & - & - & - \\
\hline $\mathbf{T i}$ & $(\mu \mathrm{g} / \mathrm{L})$ & $<1000$ & $<1000$ & - & - & $<1000$ & $<1000$ & $<1000$ & - & - & - & - \\
\hline $\mathbf{T I}$ & $(\mu \mathrm{g} / \mathrm{L})$ & $<0.13$ & $<0.13$ & $<0.01$ & $<0.01$ & $<0.13$ & $<0.13$ & $<0.13$ & $<0.01$ & $<0.01$ & $<0.01$ & $<0.01$ \\
\hline $\mathbf{U}$ & $(\mu \mathrm{g} / \mathrm{L})$ & 1.4 & 1.41 & 2.82 & 0.98 & 1.51 & 9.02 & 1.01 & 2.73 & 1.72 & 0.2 & 3.51 \\
\hline $\mathbf{V}$ & $(\mu \mathrm{g} / \mathrm{L})$ & 12.5 & 12.4 & 3 & 3.8 & 4.1 & 4.2 & $<1.3$ & 0.6 & 0.3 & 0.5 & 1.2 \\
\hline $\mathbf{W}$ & $(\mu \mathrm{g} / \mathrm{L})$ & - & - & 0.05 & 0.04 & - & - & - & 0.11 & 0.04 & 0.09 & 0.03 \\
\hline $\mathbf{Y}$ & $(\mu \mathrm{g} / \mathrm{L})$ & 0.027 & 0.017 & 0.01 & 0.01 & 0.014 & 0.042 & 0.009 & 0.05 & 0.1 & 0.01 & 0.13 \\
\hline Zn & $(\mu \mathrm{g} / \mathrm{L})$ & 30 & 25 & 11 & 11 & $<16$ & 29 & 23 & 27 & 3020 & 12 & 12 \\
\hline
\end{tabular}

(The samples LIX-1, LIX-2, GIA-10, AD-10, HL-10 analysed in Natural History museum of London labs and the rest of them in ACME labs)

XLVII, No 2 - 948 

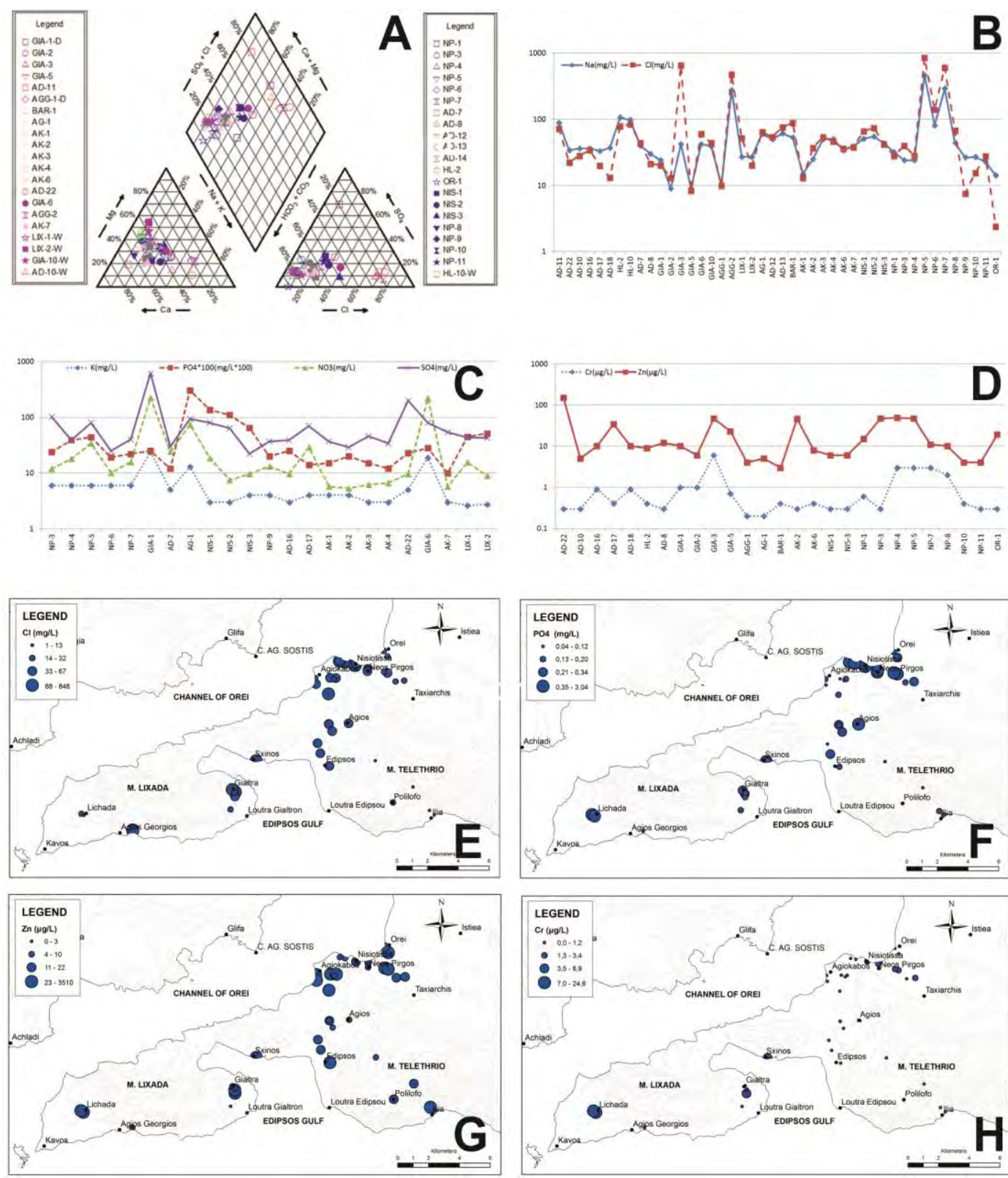

Figure 2 - (A) Chemical composition of groundwater samples plotted in Piper trilinear diagram. (B) Diagram presenting the co-varying of $\mathrm{Na}$ and $\mathrm{Cl}$ at groundwater samples. (C)

Diagram presenting the variation of $\mathrm{K}, \mathrm{NO}_{3}, \mathrm{SO}_{4}$ and $\mathrm{PO}_{4}$ at groundwater samples from lowlands, where agricultural activities take place. (D) Diagram presenting the co-varying of

$\mathrm{Cr}$ and $\mathrm{Zn}$ at groundwater samples (used only the samples in which both Fe and $\mathrm{Zn}$ concentrations are above detection limits). Geochemical maps showing the distribution of $\mathrm{Cl}$ $(\mathrm{E}), \mathrm{PO}_{4}(\mathrm{~F}), \mathrm{Zn}(\mathrm{G})$ and $\mathrm{Cr}(\mathrm{H})$ in groundwater samples.

The water samples from the peninsula of Gialtra (Sample Codes: LIX-; AGG-; GIA- and samples $\mathrm{AD}-10, \mathrm{AD}-11, \mathrm{AD}-22)$ show the maximum concentrations for a series of elements, namely $\mathrm{Cr}$ $(14.2 \mu \mathrm{g} / \mathrm{L}$; Fig. $2 \mathrm{H}), \mathrm{Ni}(6.1 \mu \mathrm{g} / \mathrm{L})$ and Fe $(35 \mu \mathrm{g} / \mathrm{L}), \mathrm{Zn}(420 \mu \mathrm{g} / \mathrm{L})$ and Co $(3 \mu \mathrm{g} / \mathrm{L})$. 
The results of chemical analysis of groundwater samples by ICP-MS (Table 3) strongly support the relationships between elemental groundwater content and rock geochemistry.

\subsection{Evaluation of Water Quality for Human Consumption}

In order to assess the suitability of the studied groundwater samples, for human consumption, the analytical values with the parametric levels, imposed by the current relative legislation (Directive 98/83/EU) were compared. It is noted that the National Greek law is in agreement with that EU Directive 98/83/EU. Even though industrial activities are absent in the studied area and only agricultural activities take place, concentrations of some chemical parameters exceeded the indicator parametric value $(17.7 \%)$ and the parametric value $(8.8 \%)$ according to Directive 98/83/EC (Tables 1 and 2).

Analytically, the Mn content of the studied groundwater samples varies from 0.09 to $260 \mu \mathrm{g} / \mathrm{L}$, while the parametric value given by Directive $98 / 83 / \mathrm{EC}$ is $50 \mu \mathrm{g} / \mathrm{L}$. This value was exceeded by three samples from the areas of Neos Pyrgos, Oreoi and Agios.

The $\mathrm{Na}$ content varies from 9 to $640 \mathrm{mg} / \mathrm{L}$, while the indicator parametric value is $200 \mathrm{mg} / \mathrm{L}$. Six samples, from Agios Georgios and Schinos of Edipsos exceed that value.

The Ca content ranges between 43 and $450 \mathrm{mg} / \mathrm{L}$, while the indicator parametric value is 100 $\mathrm{mg} / \mathrm{L}$. Four samples exceed it, from Agios Georgios and Schinos of Edipsos.

The $\mathrm{NO}_{3}$ content varies from 0.5 to $250 \mathrm{mg} / \mathrm{L}$, while the parametric value is $50 \mathrm{mg} / \mathrm{L}$. Six samples from Gialtra, Agios Georgios and Neos Pyrgos exceed it.

The $\mathrm{SO}_{4}$ content varies from 2 to $620 \mathrm{mg} / \mathrm{L}$, while the indicator parametric value is $250 \mathrm{mg} / \mathrm{L}$. Only one sample from the area of Gialtra exceeds it.

The conductivity values range between 0.4 and $7.3 \mathrm{mS} / \mathrm{cm}$, while the indicator parametric value is $2.5 \mathrm{mS} / \mathrm{cm}$. Three samples exceed it, and all these samples are from the area of Schinos of Edipsos. All the others studied chemical parameters are within the parametric values given by the Directive 98/83/EC

\subsection{Evaluation of Water Quality for Irrigation}

In order to assess the suitability of groundwaters for irrigation, their quality status was assessed through Sodium Adsorption Ratio (SAR) and Magnesium Hazard (MH). The SAR in the studied groundwater samples ranges from 0.2 to 8.6 (Table 1) which, combined with the measured values of Electrical Conductivity (EC) gave information about their classification, based on the fields suggested by U.S. Salinity Laboratory (1954). So the studies samples were classified (Table 1) as follows: S1-C1 (58\%), S1-C2 (33\%), S1-C3 (7\%) and S2-C3 (2\%). The MH (Magnesium Hazard) values, in the studied samples, ranges from 21.9 to 60.6 and $18 \%$ of the samples have $\mathrm{MH}>50$ (Table 1), meaning that they are not recommended for irrigation.

\section{Discussion - Conclusions}

The results from the study of cold groundwater, undertaken in the area of NW Euboea Greece, show that the chemical composition of the groundwater is controlled by the following three main factors: (i) the chemical composition of the local rocks (ultramafic and metamorphic), (ii) the sea, for the samples located near the seashore and (iii) the anthropogenic activities (mainly the extensive use of fertilizers and pesticides).

The results of chemical analyses through the spatial distribution of the concentrations of the various elements, show that the two main rock formations that have affected the chemical composition of the studied groundwater are the ultramafic rocks from the ophiolitic sequence as well as the metamorphic rocks occurring in the area. 
In the peninsula of Gialtra, small surface occurrences of ultramafic rocks from the ophiolithic sequence are present. The water samples from that area (Lichada, Agios Georgios, Gialtra, Schinos), show the highest concentration of $\mathrm{Cr}$ (up to $14 \mu \mathrm{g} / \mathrm{L}), \mathrm{Ni}(6.1 \mu \mathrm{g} / \mathrm{L})$ as well as high concentrations of Co (up to $3 \mu \mathrm{g} / \mathrm{L}$ ), Fe (up to $35 \mu \mathrm{g} / \mathrm{L}$ ) and $\mathrm{Zn}$ (up to $450 \mu \mathrm{g} / \mathrm{L}$ ). Enrichment of groundwater in these elements can be attributed to the impact of ultramafic rocks. Kanellopoulos (2011) and Kanellopoulos and Argyraki (2013) proved that soils and some plant species (e.g. Alyssum chalcidicum) also present high concentrations of the same elements, especially $\mathrm{Cr}$ and $\mathrm{Ni}$, even at long distances from the surface occurrences of the local ultramafic rocks.

High concentrations of $\mathrm{Zn}, \mathrm{Mg}, \mathrm{Ni}$ and to a smaller extent $\mathrm{Fe}, \mathrm{Cr}$, Co are observed near the metamorphic basement (e.g. Neos Pyrgos and Agiokampos). Enrichment of groundwater in those elements is attributed to the impact of metamorphic rocks dominating in this region. It must be noted that the soils in these areas also present high concentrations of the same elements and after geochemical and mineralogical analysis found that they have been enriched by the weathered material of the metamorphic rocks (Kanellopoulos, 2011). Of the above elements, $\mathrm{Zn}$, shows some outlier values $(>150 \mu \mathrm{g} / \mathrm{L})$ like the AK-3 sample $(3510 \mu \mathrm{g} / \mathrm{L})$. These cases may be influenced by anthropogenic sources.

The co-variation of $\mathrm{Cl}$ and $\mathrm{Na}$ suggests that they have common source. The spatial distribution of their concentrations, points out that the samples with the highest concentrations $(848 \mathrm{mg} / \mathrm{L} \mathrm{Cl} ; 642$ $\mathrm{mg} / \mathrm{L} \mathrm{Na}$ ) are from shallow aquifers near the seashore, which have been affected from the seawater and they have $\mathrm{Na}-\mathrm{Cl}$ hydrochemical type, like the case of Schinos area.

Also the co-variation of $\mathrm{K}, \mathrm{NO}_{3}, \mathrm{SO}_{4}, \mathrm{PO}_{4}$, suggests their common source. The spatial distribution of their concentrations, points out that the samples with the highest concentrations located near lowlands, where agricultural activities take place. Their source is the use of fertilizers and the variability which could be observed from area to area is related to the different degree of use of each type of fertilizer. Some samples present disproportionately high concentrations of $\mathrm{NO}_{3}$ compared to $\mathrm{SO}_{4}, \mathrm{PO}_{4}$ and $\mathrm{K}$ concentrations. These samples are within or adjacent to villages without sewerage, so the high concentration of $\mathrm{NO}_{3}$ is related to residential wastewater.

It is profound that both natural and anthropogenic factors affect the groundwater quality for human consumption to such an extent that $17.7 \%$ exceed at least one indicator parametric value and 8.8 $\%$ exceed at least one parametric value of the Directive 98/83/EC. Finally, the studied groundwater samples quality for irrigation classified based on SAR-EC as S1-C1 (58\%), S1-C2 (33\%), S1-C3 (7\%) and S2-C3 (2\%) and based on the MH (Magnesium Hazard) $18 \%$ of the samples have $\mathrm{MH}>50$ and they are not recommended for irrigation.

\section{References}

Alexander E.B., Adamson C., Zinke P.J. and Graham R.C, 1989. Soils and conifer forest productivity on serpentinized peridotite of the Trinity Ophiolite, California, Soil Science, $148(6), 412-423$.

Aubouin J. 1959. Contribution a l'étude géologique 'de la Grèce septentrionale: Les confins de l'Epire et de la Thessalie, Ann. Géol. Pays Hellen, 10, 1-483.

Brooks R.R. 1987. Serpentine and its vegetation: Portland, OR, Dioscorides Press.

Directive 98/83/EU. On the quality of water intended for human consumption.

Gasser U.G. and Dahlgren R.A. 1994. Solid-phase speciation and surface association of metals in serpentinitic soils, Soil Science, 158, 409-420.

Georgalas G.C. 1938. Le volcans des $1^{\wedge}$ les Likhades et de Hagios Ioannis (Kammena Vourla), Praktika Academia Athinion, 13, 86-98.

Gioni-Stavropoulou G. 1983. Inventory of hot and mineral springs of Greece, I, Aegean sea, Hydrological and Hydrogeological Investigation Report, No. 39, IGME, Athens (in Greek). 
Gough L.P., Meadows G.R., Jackson L.L. and Dudka S., 1989. Biogeochemistry of a highly serpentinized, chromite-rich ultramafic area, Tehama County, California, U.S. Geological Survey Bulletin, 1901, 1-24.

Kabata-Pendias A. and Mukherjee A. 2007. Trace Elements from Soil to Human. Springer-Verlag, Berlin, New York.

Kabata-Pendias A. 1995. Agricultural problems related to excessive trace metal contents in soils, in: Salomons, W., Forstner, U., Mader, P. (Eds.), Heavy Metals: Problems and Solutions. Springer-Verlag, Berlin Heidelberg, pp. 3-18.

Kanellopoulos C. 2006. Geochemical research on the distribution of metallic and other elements to the groundwater in Fthiotida Prefecture and N. Euboea. Master Thesis, University of Athens, Greece (in Greek).

Kanellopoulos C. 2011. Geochemical research on the distribution of metallic and other elements to the cold and thermal groundwater, soils and plants in Fthiotida Prefecture and N. Euboea. Environmental impact, Ph.D. Thesis, University of Athens, Greece (in Greek).

Kanellopoulos C. 2012. Distribution, lithotypes and mineralogical study of newly formed thermogenic travertines in Northern Euboea and Eastern Central Greece, Central European Geosciences, 4(4), 545-560.

Kanellopoulos C. and Argyraki A. 2013. Soil baseline geochemistry and plant response in areas of complex geology. Application to NW Euboea, Greece, Chemie der Erde - Geochemistry, doi: 10.1016/j.chemer.2013.06.006

Le Pichon X. and Angelier J. 1979. The Hellenic Arc and Trench system: A key to the neotectonic evolution of the Eastern Mediterranean area, Tectonophysics, 60, 1-42.

McKenzie D. 1970. Plate Tectonics of the Mediterranean Region, Nature, 226, 239-242.

McKenzie D. 1972. Active tectonics of the Alpide - Himalayan belt: the Aegean Sea and surrounding regions (Tectonics of the Aegean Region), Geophys. J. Roy. Astr. Soc., 55, 217-254.

Mountrakis D. 1986. The Pelagonian zone in Greece: A polyphase deformed fragment of the Cimmerian continent and its role in the geotectonic evolution of the Eastern Mediterranean, Journal of Geology, 94, 335 - 347.

Mueller M. 1994. Distribution of cadmium in the food chain (soil-plant-human) of a cadmium exposed area and the health risks of the general population, Sci. Total Environ., 156, 151158.

Needleman H.L. 1980. Low Level Lead Exposure-The Clinical Implications of Current Research. Raven Press, New York.

Oze C., LaForce M., Wentworth C., Hanson R., Bird D. and Coleman R. 2003. Chromium geochemistry of serpentinous sediment in the Willow core, Santa Clara County, CA. U.S. Department of the interior, U.S. geological survey, Open-File Report 03-251.

Pe-Piper G. and Piper D. 2002. The igneous rocks of Greece, the anatomy of an orogen, Gebruder Borntraeger, Berlin.

Richards L.A. 1954. Diagnosis and improvement of saline and alkali soils, Agriculture handbook, Volume 60, US Department of Agriculture, Washington DC.

Schreier H., Omueti J.A. and Lavkulich L.M. 1987. Weathering processes of asbestos-rich serpentinitic sediments, Soil Science Society of America Journal, 51, 993-999.

Szabolcs I. and Darab C. 1964. The influence of irrigation water of high sodium carbonate content of soils. In: Proceedings of 8th international congress of ISSS, Transaction II, 803-81.

U.S. Salinity Laboratory 1954. Diagnosis and improvement of saline and alkali soils. In Richards L. A. (Ed.), USDA agriculture handbook No. 60. Washington, D.C.: U.S. Government Printing Office.

Vavassis I. 2001. Geology of the Pelagonian zone in Northern Evia Island (Greece): Implications for the geodynamic evolution of the Hellenides. These de doctorat, Univ. de Lausanne. 